\title{
No normative free lunch: relevance and the autonomy of the normative domain
}

\section{Singa Behrens ${ }^{1}$ (iD}

Received: 16 November 2020 / Accepted: 17 August 2021 / Published online: 30 August 2021

(c) The Author(s) 2021

\begin{abstract}
The autonomy thesis is the claim that one cannot get a normative statement from purely descriptive statements. But despite its intuitive appeal a precise formulation of the thesis has remained elusive. In a recent paper, Maguire (2015) makes the promising suggestion that the thesis should be understood in terms of ground. But Maguire's formulation, I argue, is based on controversial taxonomic assumptions that make the autonomy thesis into a non-substantive claim. I develop an alternative ground-based formulation of the autonomy thesis that appeals to the notion of normative relevance, which is in turn understood using the tools of truthmaker semantics. This formulation of the autonomy thesis avoids well-known counterexamples to other formulations and has significant advantages over Maguire's formulation.
\end{abstract}

Keywords Autonomy · Is-Ought gap · Relevance · Ground · Truthmaker semantics

\section{Introduction}

What is the relationship between the ethical domain —or more generally, the normative domain ${ }^{1}$ - and the descriptive domain? The normative domain seems to be based, at least in part, in the descriptive domain. For instance, that it is morally required to help a drowning child is at least partly explained by descriptive features of the act of life-saving, such as this act's avoiding harm to the child. But many philosophers have

\footnotetext{
1 One might think that the autonomy debate should be restricted to the ethical domain. I assume that as far as the formulation of an autonomy thesis is concerned the debate generalizes to the normative domain. Things might need further qualification, if one aims at a defense of the autonomy thesis.
}

$凶$ Singa Behrens

singa.behrens@uni-hamburg.de

1 Department of Philosophy, University of Hamburg, Überseering 35, 22297 Hamburg, Germany 
thought there is nevertheless a sense in which the normative domain is autonomous ${ }^{2}$ from the descriptive domain - a sense in which the descriptive domain does not suffice to generate the normative domain.

The autonomy thesis in its most abstract form states that we cannot get a normative statement from purely descriptive statements. The autonomy debate is concerned with the question of how this abstract claim should be made precise and whether the precise formulations are true. Any concrete version of the abstract autonomy thesis must answer at least three questions.

Q1 What are descriptive statements?

Q2 What are normative statements?

Q3 What does it mean to say that we cannot get statements of kind $A$ from statements of kind $B$ ?

The aim of this paper is to develop a new explication of the abstract autonomy claim that satisfies the following desiderata: ${ }^{3}$ The autonomy thesis

(D1) explicates a sense of autonomy such that autonomy is a feature of the nature of normativity;

(D2) is not falsified by spurious counterexamples;

(D3) is sensitive to, i.e. falsifiable by, genuine counterexamples;

(D4) is falsified if certain metaethical views, according to which the normative domain is identical with or reducible to the descriptive domain, are true, and

(D5) entails a non-trivial divide of the metaethical terrain.

To get a better understanding of the sense of autonomy that we are concerned with, let us go through the desiderata and see why they impose reasonable requirements on what I shall call a substantial sense of autonomy. Note that there are several ways to interpret the abstract autonomy claim. One example is an interpretation in logical terms according to which there is no strict entailment from descriptive claims to normative claims. ${ }^{4}$ Another is the Moorean one according to which normative concepts cannot be analyzed in descriptive terms. A third is a metaphysical interpretation according to which normative facts seem different in kind from descriptive facts. ${ }^{5}$ None of these interpretations captures exactly the sense of autonomy that we are concerned with. ${ }^{6}$ For we are interested in a sense of autonomy that concerns explanatory connections between the descriptive and the normative domain.

Now, the first desideratum, (D1), states that the autonomy thesis must explicate a sense of autonomy that concerns the nature of normativity. ${ }^{7}$ This requirement is supposed to rule out explications of autonomy that turn it into a general feature of any separable class of statements. So for example Pigden (1989) famously argued that

\footnotetext{
2 This particular technical use of the term originates from Rynin (1957).

3 Thanks to an anonymous referee for pushing me to clarify this point.

4 See e.g. Pigden (2010) for several different logical autonomy theses. The logical autonomy debate is connected to the Is-Ought problem famously articulated by Hume (1978, p. 469).

5 See for example (Enoch 2010).

6 Note that the autonomy thesis that we are concerned with might be compatible with each of the interpretations.

7 See e.g. (Maguire 2015, p. 199), and (Bader 2017, p. 112) for similar considerations.
} 
the autonomy of the normative domain is an instance of a more general property of logical conservativeness. As a result, the domain of bird-statements, i.e. statements that involve bird-vocabulary, is autonomous with respect to the non-bird domain, i.e. statements that do not involve bird-vocabulary. But intuitively, autonomy is-even if not a unique - a special feature of the normative domain. If the bird-domain is autonomous at all, then it is so in a quite different sense from what we take to be at issue in the normative case. We are concerned with an autonomy thesis that teaches us something about the nature of normativity.

The first desideratum is related to the two subsequent desiderata (D2) and (D3). First, I clarify the distinction between spurious and genuine counterexamples. ${ }^{8}$ Genuine counterexamples and their potential to falsify autonomy theses emerge from (some of) the involved normative components and their close relation to the descriptive domain. So if normativity is non-autonomous, then the nature of normativity is intimately connected to the descriptive realm. A paradigm example would be a case where descriptive facts about speech-acts like promises suffice to generate facts about obligations. Another would be a case where facts about goodness would be (multiply) realized by purely descriptive facts about, e.g. the promotion of friendship. In contrast, spurious counterexamples emerge from merely formal features. Spurious counterexamples hold independently of the nature of normativity. A paradigm example is the disjunctive case in Prior (1960). Consider a disjunction $D \vee N$ that has a normative disjunct $N$ and a descriptive disjunct $D$. It is assumed that the disjunction is either normative or descriptive. In either case, a normative conclusion is entailed by descriptive premises. For if the disjunction is normative, then it is entailed by descriptive $D$. On the other hand, if it is descriptive, then it together with plausibly descriptive $\neg D$ entails normative $N$. Note that the falsifying potential of the example does not emerge from the normative parts it contains. Another spurious counterexample makes use of the descriptive statement that Sarah does not exist and the normative statement that there is no obligation for Sarah to donate. Again, the example seems to result from general formal features rather than features of normativity. Autonomy theses that are falsified by spurious counterexamples do not teach us anything about normativity. On the other hand, theses that are immune to genuine counterexample seem to be trivial. So (D2) and (D3) are reasonable desiderata.

The fourth desideratum (D4) is in the same vein. Metaethical views according to which the normative domain is identical with or reducible to the descriptive domain plausibly deny that when it comes to explanatory connections between the normative and the descriptive domain normativity by its nature possesses autonomy. In other words, the descriptive domain would suffice for explanations of normative matters. Thus, an autonomy thesis must be incompatible with these views. Last, we already stated that some metaethical views must be incompatible with a substantial autonomy thesis. Yet, it is a further desideratum, (D5), that some metaethical views can accept the autonomy thesis and what is more, an autonomy thesis is not supposed to be an explication of one particular metaethical position. Thus, we require a non-trivial divide of the metaethical terrain.

\footnotetext{
8 See e.g. Prior (1960), Pigden (1989), and Fine (2021) for similar distinctions.
} 
In what follows I develop a ground-based autonomy thesis that provides us with such a substantial understanding of what it is for the normative domain to be autonomous. A metaphysical version of the autonomy thesis along ground-theoretic lines was first suggested by Maguire (2015). To a first approximation, one might take the autonomy thesis to be the claim that no descriptive statements ground any normative statements. But spurious counterexamples show that this will not work. The crucial feature of the counterexamples is that the descriptive grounds are not relevant to what I will call the normative parts of the grounded statement. So instead, we should understand the autonomy thesis in terms of normatively relevant grounding: No normative proposition is grounded in descriptive propositions in a normatively relevant way. As we will see, this relevant autonomy thesis satisfies the previous desiderata.

Here is the outline of the paper: first, I will present answers to the first and second questions, Q1 and Q2, within truthmaker-based semantics in Sect. 2. In Sect. 3, I return to the third question in more detail. I will introduce the ground-theoretic approach developed in Maguire (2015) and raise concerns for this approach. Thereafter, I will interpret the autonomy thesis in terms of normatively relevant grounding. In Sect. 4, I argue that the resulting relevant autonomy thesis satisfies the desiderata. In Sect. 5, I will highlight a number of ways in which my proposal improves on Maguire's approach. I conclude in Sect. 6.

\section{The normative and the descriptive domain}

There are several choices to be made when we determine the normative and the descriptive domain to which an autonomy thesis applies. The first qualification depends on the project that we pursue. If we pursue an epistemic project, then we might consider statements by persons or belief states and their justification. If we pursue a logical project, we might consider linguistic entities like normative sentences or propositions. In this paper, I suggest a ground-theoretic interpretation of the autonomy thesis. Within the grounding debate, grounding has been interpreted along metaphysical lines and along representational lines. ${ }^{9}$ Metaphysical interpretations focus on facts that are taken to be worldly items. On the other hand, representational interpretations focus on propositions. This distinction especially matters to the granularity of grounding claims because usually the individuation of propositions is more fine-grained. For the moment, I will stay neutral with respect to this distinction. While I will consider grounding to be a relation among propositions rather than facts, this assumption will ultimately be compatible with a worldly conception of grounding. However, one advantage of the focus on propositions is that our initial formulation of the autonomy thesis does not presuppose normative realism, i.e. the existence of normative facts. ${ }^{10}$ Furthermore, we avoid general doubts concerning negative or disjunctive facts. Given the restriction to propositions, we can reformulate the first two questions as follows:

\footnotetext{
9 See Trogdon (2013) for an overview.

10 The taxonomy that we impose upon propositions will be available for normative anti-realists provided that they accept minimalism about truth-aptness and propositions. According to minimalism, disquotational schemas like ' $S$ ' is true iff $S$ entail everything there is to know about e.g. truth. For a helpful discussion of minimalism in metaethics, see (Asay 2013).
} 
Q1* What is a descriptive proposition?

$\mathrm{Q} 2 *$ What is a normative proposition?

To answer these questions some criteria are necessary that divide the domain of propositions into at least two domains. I will assume that the descriptive-normative distinction is exclusive and exhaustive. That is, every proposition is either normative or descriptive but not both. To assume exclusivity on the semantical level does not prejudge metaphysical questions of whether the normative domain can ultimately be reduced to the descriptive domain. So it is consistent with it being a merely representational matter whether a proposition is normative or descriptive. The reason to assume exhaustivity is that a defense of a strong autonomy thesis needs to establish the autonomy of a particular domain within the domain of all propositions. So, the claim is that by singling out the normative propositions, we get a domain of propositions that is autonomous with respect to the remaining ones. I take the descriptive domain to be the complement of the normative domain. Otherwise, weaker claims would result from accepting propositions that are neither descriptive nor normative. ${ }^{11}$ The resulting thesis would only establish a restricted autonomy thesis relative to particular subdomains. For example, Oddie (2018) introduces a logical autonomy thesis that does not apply to mixed disjunctions: No collection of purely natural premises entails a purely normative proposition. ${ }^{12}$ Note that even if this assumption is dropped, the result of the paper can be maintained by restricting the scope of the autonomy thesis to suitable subdomains.

It is a plausible assumption that we have an intuitive grasp on the descriptivenormative distinction at least in several cases. Compare the following two propositions.

Sarah gives five percent of her income to poor people.

Sarah is morally obliged to give five percent of her income to poor people.

The first proposition is only about the way the world actually is and the second one is also about how the world ought to be. If the state that Sarah gives five percent of her income to poor people obtains, then the first proposition is true. The obtaining of this state is neither necessary nor sufficient for the truth of the second proposition. For the second proposition to be true, the world must be such that a normative state obtains that concerns Sarah's obligations. ${ }^{13}$ The descriptive-normative distinction that I will offer is based on this observation. Note that to make the distinction between normative and descriptive propositions precise a fine-grained view of propositions is required. Consider the proposition that Sarah ought to donate or that it is not the case that she ought to donate. This proposition is arguably normative, since there is a normative state that makes this proposition true. This truth is necessarily equivalent to e.g. mathematical truths that are plausibly descriptive. An intensional framework, i.e. a framework that is only sensible to differences in the extension of proposition across modal space, would identify necessarily equivalent propositions with each other.

\footnotetext{
11 One option is to count disjunctions with a normative and a descriptive disjunct to be neither descriptive nor normative. See e.g. Oddie (2018) who discusses natural-normative hybrids.

12 See (Brown 2014) for a critical discussion of the no-mixed-sentences-strategy.

13 The talk of states in the world need not be taken with the metaphysical seriousness that might worry anti-realists. I will return to the assumption of states in our semantics and in our ontology in due course.
} 
Thus, either both of them are descriptive or both of them are normative. An intensional framework is thus too coarse-grained to make the relevant distinctions. ${ }^{14}$ I start by introducing truthmaker semantics to offer a hyperintensional view of propositions and proceed by presenting the truthmaker-based criteria for a descriptive-normative distinction.

\subsection{Propositions and truthmakers}

The account adopts a fine-grained view of propositions. An intensional approach only distinguishes between two propositions, if there is at least one possible world where the propositions differ in truth value. Accordingly, all necessary truths express the same proposition and more generally all necessarily equivalent propositions are identified with each other. Intensional semantics are too coarse-grained to make the relevant distinction between normative and descriptive propositions. The truthmaker approach as developed by Kit Fine in a recent series of publications introduces a hyperintensional framework that allows for more fine-grained distinctions. In what follows, I will give a brief introduction to the Finean framework, which is introduced in more detail in Fine (2017a,b).

A proposition is identified with the set of all of its truthmakers. Roughly, a truthmaker of a proposition is a state or a situation that would make the proposition true, if it were to obtain. It is important to note that the term 'state' that we use is a term of art and need not correspond to an intuitive sense of the term. However, possible states can be thought of as fragments or proper parts of possible worlds, like the state that it is sunny or the state that Socrates exists. Furthermore, we allow for impossible or inconsistent states like the state of it being hot and cold. An important feature of the truthmaking relation is that it is supposed to be exact. That is, the truthmaker of a proposition must be relevant as a whole to its truth. This means that if we add an arbitrary state to a truthmaker of a proposition, then the result is not guaranteed to be a truthmaker of the proposition in question. Furthermore, like possible worlds, states are required to be non-disjunctive, or determinate. ${ }^{15}$ Consider the proposition that it is rainy or sunny today and its truthmakers. One truthmaker is the presence of sun, another is the presence of rain, but there are not in addition to that any disjunctive truthmakers. There is no state like the presence-of-rain-or-the-presence-of-sun that makes the disjunction true.

Another important feature of states is that they stand in mereological relationships to each other. The state $h$ that it is hot is a part of the more complex state that it is hot and sunny. I will denote the parthood relation among states by $\sqsubset$. We assume that any states $s_{1}, s_{2}, \ldots$ have a fusion $s$. The fusion is supposed to be the smallest state that has all of $s_{1}, s_{2}, \ldots$ as parts. We denote the fact that $s$ is the fusion of $s_{1}, s_{2}, \ldots$ as follows: $s=s_{1} \sqcup s_{2} \sqcup \ldots$..

Within this framework, we can define some of the Boolean operations like conjunction and disjunction.

[1] $P \wedge Q=\{p \sqcup q: p \in P$ and $q \in Q\}$

\footnotetext{
14 See (Fine 2021).

15 This is standardly assumed within truthmaker semantics. See (Fine 2017a).
} 
[2] $P \vee Q=\{s: s \in P \text { or } s \in Q\}^{16}$

A state $s$ makes the conjunction $P \wedge Q$ true iff $s$ is the fusion of a truthmaker of $P$ and a truthmaker of $Q$. A state $s$ makes the disjunction $P \vee Q$ true iff it is either a truthmaker for $P$ or a truthmaker for $Q$. In the case of a disjunction, we might prefer to work with the closure under fusion of $\{s: s \in P$ or $s \in Q\}$. The closure under fusion is the smallest set that includes all the fusions of the truthmakers in $\{s: s \in P$ or $s \in Q\}$. To deal with the negation of a proposition, $\neg P$, we need to countenance falsemakers of propositions as well as their truthmakers. The proposition that it is not the case that it is rainy today is plausibly made true by those states that make the proposition that it is rainy today false. For now, negation may be set aside, and thus so may falsemakers.

\subsection{The descriptive-normative distinction: an asymmetry}

This section addresses the questions $\mathrm{Q} 1 *$ and $\mathrm{Q} 2 *$. We have defined propositions in terms of sets of states. This suggests a descriptive-normative distinction in terms of a distinction between descriptive and normative states. Descriptive propositions have descriptive truthmakers and normative propositions have normative truthmakers. One might worry that a distinction between normative and descriptive propositions in terms of the worldly states making them true is adequate only assuming realism about the normative domain. In response, following Fine (2021) (and Jago (2020)), I will assume that we need not regard the assumption of normative states as metaphysically significant in this way. Arguably, the way we consider truthmakers in our semantics can be treated separately from metaphysical assumptions. ${ }^{17}$ For now, I will show that the distinction allows us to give a precise formulation of the autonomy thesis that avoids the potential counterexamples under discussion. One advantage of the focus on truthmakers is that by their specificity we can handle problems that emerge from disjunctions.

There is an asymmetry between the normative and the descriptive domain: ${ }^{18}$ The descriptive is pure in the sense that it does not contain any normative parts. In contrast, the normative is not pure in the corresponding sense. It can contain descriptive parts. To see why, consider Sarah's obligation to donate. Plausibly there are several descriptive states that underly this normative state, among them for example Sarah's existence and states to the effect that Sarah can donate. The following taxonomy that was introduced by Fine (2021) accounts for this asymmetry.

We take the distinction between normative and descriptive states as primitive. Yet we introduce constraints on their interrelation. First, we assume a non-empty set of descriptive states $S^{D}$ that is closed under part and closed under fusion. This assumption entails that every descriptive state is purely descriptive in the sense that it can only

\footnotetext{
16 [2] requires the relative specificity of states that was mentioned earlier.

17 Again, the taxonomy is thereby available to anti-realists who accept minimalism about propositions and truthmakers. See footnote 10 .

18 See e.g. Rosen (2017). See also the debate about thick and thin normative/ethical concepts introduced by Williams (1985).
} 
contain descriptive parts, and it entails that any state that can be obtained by fusing only descriptive states will itself be descriptive. ${ }^{19}$

Second, we assume that the set of normative states $S^{N}$-i.e. the set of all states not in $S^{D}$ —is also non-empty. Note that by the assumptions about $S^{D}, S^{N}$ will be upwardsclosed, so any state containing a normative state as part will also be normative. We thereby adopt a broad concept of normative states. However, we can make interesting distinctions between different degrees of purity with respect to the normative states. I will return to a narrower concept of normative states later in the paper.

For the present taxonomy, to have a normative truthmaker of any kind is the demarcation line between descriptive and normative propositions. If there is at least one way for a proposition to be true that requires some normative state to obtain, then this proposition is part of the normative domain. This is a broad notion of the normative domain because it only requires the existence of at least one normative truthmaker for a proposition to be normative. ${ }^{20}$ A descriptive proposition has only descriptive truthmakers. Now we are in the position to answer Q1* and Q2*:

A1* A descriptive proposition is a set of descriptive states.

A2* A normative proposition is a set of states that contains at least one normative state.

The definitions of the descriptive and the normative domain is sensible to hyperintensional distinctions. Moreover, the taxonomy of normative and descriptive content is independently motivated. Hence, it does not depend on the truth of the autonomy thesis.

\section{Ground-based autonomy theses}

Now we can turn to the third question Q3. I have already pointed out that I take the autonomy thesis to be a ground-theoretic claim. An interpretation of the autonomy thesis along these lines was first suggested by Maguire (2015). Grounding requires an intimate connection between the grounded proposition on the one hand and the grounds on the other hand that is characterized by explanatory relevance. ${ }^{21}$ Some accounts try to explain away potential counterexamples to logical autonomy theses by appealing to relevance considerations. ${ }^{22}$ While the details of the diagnosis differ, the concept of relevance appears to be crucial. I lack the space to discuss in detail whether there

\footnotetext{
19 Largely as a matter of technical convenience, it is standardly assumed within truthmaker semantics that there exists a null-state, which is by definition part of every state. By the assumption of closure under part, the null-state is descriptive. Note that this means that every normative state has at least the null-state as a descriptive part.

20 This definition is sensible only under an exact conception of truthmaking. If we merely appealed to a monotonic form of truthmaking, i.e. a form of truthmaking where we can fuse an arbitrary state to a truthmaker and the fusion is still a truthmaker, every proposition would have a normative truthmaker.

21 Formulating the autonomy claim in terms of ground highlights connections between the autonomy debate and the debate between ethical naturalism and non-naturalism as it is characterized in Rosen (2017). We will locate ground-theoretic autonomy theses within the general metaethical terrain in more detail towards the end of the paper.

22 For example Pigden (1989), and Singer (2015). I cannot discuss them at length here. However, I will briefly sketch a worry concerning their accounts. The accounts classify propositions or sentences as
} 
are successful logical autonomy theses and to compare them with a ground-theoretic approach. My primary aim is to show that there is a ground-theoretic interpretation that makes a substantial claim in the sense specified by the desiderata and that avoids implausible taxonomic consequences. For this purpose, I will briefly discuss a simple ground-theoretic autonomy thesis and argue that a restriction of the normative domain is not satisfying. Thereafter, I will introduce Maguire's ground-theoretic approach and show that it is subject to substantial concerns. Finally, I will introduce the relevant autonomy thesis and argue that it avoids those problems.

\subsection{Simple ground-theoretic approach}

According to the simple ground-theoretic autonomy thesis no normative proposition is fully grounded by descriptive propositions. However, this interpretation is subject to spurious counterexamples. Examples that are familiar from the logical debate falsify the simple ground-theoretic autonomy thesis:

DISJUNCTION: We start with a descriptive proposition $D$ and a normative proposition $N$. The disjunction $D \vee N$ is normative because it has at least one normative truthmaker. By a commonly assumed grounding principle, each of the true disjuncts grounds the disjunction. So, descriptive $D$ grounds normative $D \vee N .^{23}$

ABSENCE: Consider the descriptive proposition Sarah does not exist. This proposition arguably grounds the plausibly normative proposition There is no obligation for Sarah to donate. ${ }^{24}$

One strategy to secure the simple ground-theoretic autonomy thesis is to restrict the normative domain. This strategy has been pursued in the logical autonomy debate. ${ }^{25}$ According to this strategy, mixed propositions like $D \vee N$ are not genuinely normative. The simple autonomy thesis only applies to the genuine normative domain. It is noteworthy that the truthmaker framework can be used to make this distinction precise. There are distinctions between different degrees of purity with respect to the normative states. ${ }^{26}$ We can distinguish between broadly normative states and strictly normative states. Broadly normative states can contain descriptive states as additional parts that are simply added to a normative state by fusion. Any fusion of a normative state and a descriptive state is a broadly normative state. The state that Sarah is obliged to donate and John exists, $n \sqcup d$, is a broadly normative state. In contrast, strictly nor-

Footnote 22 continued

relevantly normative relative to the argument in which they figure as conclusions. We will see in the discussion of Maguire that an argument-relative strategy is subject to the worry that it does not make a claim about the nature of the normative domain. Pigden (1989) endorses this result.

23 Recall that initially Prior introduces this example in the form of a dilemma. A ground-theoretic autonomy thesis is not subject to the second horn of the dilemma, since $\neg D$ and $D \vee N$ do not ground $N$. So, if $D \vee N$ turns out to be descriptive, there is no problem for a ground-theoretic autonomy thesis.

24 This example is a slightly modified version of the example in (Fine 2021, p. 897).

25 For example Gibbard (2012) and Russell and Restall (2010).

26 For our purposes it will suffice to distinguish between broadly and strictly normative states. For a more detailed distinction see (Fine 2021). 
mative states contain all their descriptive parts as integral parts. Intuitively, an integral part of state $s$ is such that we cannot obtain $s$ by glueing together the integral part and another proper part of $s$. The integral part cannot be supplemented with a proper part of $s$ to obtain $s$. One example is the state that Sarah ought to donate. This state contains the descriptive state that Sarah exists. But there is plausibly no proper part of the state that Sarah ought to donate that we can add to her existence such that the state that she ought to donate obtains. While broadly normative states can be separated into a descriptive component and a normative component, this does not hold for strictly normative states.

This distinction can be used to exclude mixed disjunctions - or more general mixed propositions-by modifying the definition of the normative domain. One example is to require that a normative proposition has only strictly normative truthmakers. Since $D \vee N$ is made true by $d \sqcup n$, which is a broadly normative state, $D \vee N$ would be non-normative.

However, excluding mixed propositions from the normative domain in general might be problematic. I will list three cases that raise doubts concerning this strategy. First, consider the proposition that there is no obligation for Sarah to donate. This is intuitively a normative proposition, but there is at least one descriptive truthmaker, namely the state that Sarah does not exist. Second, assume that a thick normative concept like selfish can be analyzed in terms of being morally wrong in a certain way and giving some significant degree of priority to oneself over others. Plausibly, under this assumption the proposition that Sarah is selfish has the same truthmakers as the conjunction that Sarah behaves morally wrong in a certain way and gives some significant degree of priority to herself over others. This conjunction is a mixed proposition. Propositions that include thick normative concepts should not be excluded from the normative domain. Third, some conditionals are arguably normative like e.g. that if nonhuman animals are automata, it is ethically permissible to kill them. ${ }^{27}$ Though it is an open question which states make conditionals true, one plausible candidate in this case is a state to the effect that nonhuman animals are automata and it is ethically permissible to kill automata, which is a broadly normative state. Moreover, some intuitively normative conditionals like that if all Church officers ought to be reverent, then all undertakers ought to be might have purely descriptive truthmakers like the state that all undertakers are Church officers. Thus, under a narrower conception of the normative, these conditionals are non-normative.

Generally speaking, we may distinguish between two different strategies for dealing with potential counterexamples. One way adopts a narrower conception of the normative domain and the other way adopts a narrower conception of what it means to get normative propositions from descriptive propositions. I will pursue the second strategy and argue that relevance plays an important role in understanding what it means to get a normative proposition from descriptive propositions. ${ }^{28}$ Before turning to my proposal, I will discuss Maguire's solution to the problem.

\footnotetext{
27 See e.g. Clipsham (2015).

28 Although I will pursue the second strategy, there is an equivalent formulation of my proposal that adopts a narrower conception of the normative domain. It contains all propositions that have only normative truthmakers. That excludes mixed disjunctions but includes mixed conjunctions.
} 


\subsection{Maguire's ground-theoretic approach}

Maguire (2015) argues that the focus on logical interpretations of the autonomy thesis is misguided. He proposes a ground-theoretic interpretation of the autonomy thesis. I start by presenting his autonomy thesis. I then argue that Maguire's autonomy thesis is subject to serious objections that render it less convincing as a substantial autonomy thesis. I introduce worries concerning the taxonomic consequences and the informative value of Maguire's thesis.

Maguire's autonomy thesis focuses on facts which he takes to be worldly items. ${ }^{29}$ Note however that within Maguire's account facts are true propositions. One important thing to notice about the way Maguire presents his autonomy thesis is that the two questions that concern the normative-descriptive distinction and the third question that concerns the way autonomy is understood are not addressed separately. While this strategy is not worrisome in itself, we will see that it invites the worries that I will point out. Maguire's worldly autonomy thesis includes two separate parts (Maguire 2015, pp. 194-195):

Metaphysical Autonomy (MA):

Converse Metaphysical Autonomy (CMA): Any fact partly ${ }^{31}$ grounded by a normative fact is a normative fact. ${ }^{32}$

Maguire's autonomy thesis does not presuppose an independent taxonomy of facts. However, he assumes an intuitive grasp on paradigmatic cases of both descriptive and normative facts. His autonomy thesis is supposed to determine the taxonomic status of non-paradigmatic cases. I will discuss DISJUNCTION to illustrate how Maguire's autonomy thesis works. I will refer to the fact expressed by a sentence $P$ by enclosing it in square-brackets. Assume $[D]$ and $[N]$ are paradigmatic cases of a descriptive and a normative fact, respectively. According to common assumptions about grounding disjunctions, there are three schematic ways to ground the disjunctive fact $[D \vee N]$ : (i) solely by $[N]$, (ii) solely by $[D]$, and (iii) by $[D]$ and $[N]$ together. ${ }^{33}$ In the first case, since $[N]$ is normative the disjunctive fact is normative by CMA. The example is compatible with MA because the sole ground of $[D \vee N]$ is normative. The third case is similar since here $[D \vee N]$ is partly grounded by $[N]$. So again, $[D \vee N]$ is normative by CMA and compatible with MA because one of its grounds is normative. In the second case, $[D \vee N]$ is descriptive because $[D \vee N]$ is grounded just by the descriptive fact $[D]$ and hence, according to MA, cannot be normative. In the third case there is a descriptive fact that is grounded by a descriptive fact. This result is compatible with MA. By distinguishing these cases, Maguire can show that the example does

\footnotetext{
29 See (Maguire 2015, p. 196).

30 Maguire uses a different terminology. He refers to ethical and non-ethical facts instead. He distinguishes between non-ethical facts and facts that are not ethical. According to his terminology the domain of nonethical facts and ethical facts overlap (Maguire 2015, p. 200, fn.26). To avoid a misleading terminology, I will refer to descriptive facts instead of non-ethical facts and to normative facts instead of ethical facts.

31 A fact $\alpha$ partly grounds a fact $\beta$ iff there is a collection of facts $\Gamma$ including $\alpha$ that grounds $\beta$.

32 For criticism of CMA see Woods (manuscript).

33 This is standardly assumed and entailed by Fine (2012a)'s elimination rules for grounding.
} 
not contradict his autonomy thesis. However, I will argue that this strategy entails implausible results. The example will help to illustrate the worries that concern this version of the autonomy thesis.

The Problem of Contingentism. This worry concerns the taxonomic consequences of Maguire's autonomy thesis. This is important, since his metaphysical autonomy thesis incorporates two functions of specifying the abstract autonomy claim and the descriptive-normative distinction. Accordingly, its plausibility partly depends on the acceptability of the resulting distinction. Most notably, Maguire's autonomy thesis entails that the taxonomic status of facts (more precisely, instances of fact-types) is contingent and depends on the actual grounds of the fact. We already saw that the disjunctive fact $[D \vee N]$ can be normative or descriptive depending on whether $[N]$ obtains. It also can be both normative and descriptive if both disjuncts obtain. ${ }^{34}$ Consequently, Maguire's autonomy thesis is committed to contingentism concerning the taxonomic status of facts.

Now the worry is that this contingentism entails implausible taxonomic consequences. Recall that Maguire assumes that at least in some cases we have an intuitive grasp of the taxonomic status of facts. Consider the following two disjunctive facts: [it is raining or murder is morally wrong] and [it is raining or charity is morally wrong]. If it rains and murder is wrong while charity is not, then the first fact is normative while the second one is not. Granted that the taxonomic status of disjunctive facts is not trivially accessible, but it seems to be implausible that the two facts belong to different categories concerning their normative status. Both disjunctive facts seem to be partly about rainy weather and partly about moral wrongness. As Woods (manuscript) puts it:

It would be a radical move to deny that the status of a fact as ethical or natural is largely due to what the fact is about, especially because the intuition driving MA relied on there being a difference in subject matter between the clearly ethical and the clearly natural facts (Woods manuscript, p. 19).

A related aspect concerns non-obtaining facts, i.e. false propositions. They are not subject to Maguire's autonomy thesis at all. The present form of contingentism seems to render us implausibly ignorant of the taxonomic status of false propositions. ${ }^{35}$ Maguire discusses this issue and objects that his account is supposed to be concerned with facts rather than propositions in general. Yet, we took the autonomy thesis to concern the nature of the normative and hence the nature of e.g. normative properties. The result that the taxonomy is dependent on the actual truth of propositions does not seem to be well motivated and moreover does not seem to fit the initial motivation of the autonomy claim.

One option to avoid these unintuitive results and to offer a more general account is to understand MA and CMA in terms of non-factive grounding. This is to say that the grounds and the grounded need not obtain in order to correspond true grounding

\footnotetext{
34 At least, this is what Maguire seems to be sympathetic to (Maguire 2015, p. 200, fn.26).

35 A similar objection is raised by Maitzen (2010): Since we do not know whether Goldbach's Conjecture is true, we do not know the taxonomic status of those conjunctions and disjunctions that involve it.
} 
claims. ${ }^{36}$ If MA and CMA are understood along these lines, [it is raining or murder is morally wrong] and [it is raining or charity is morally wrong] would both be normative according to CMA. However, note that we would have a normative proposition that is fully grounded in a descriptive proposition. Maguire himself mentions what he calls counterfactual grounding and offers different versions of a general taxonomy of propositions in terms of counterfactual grounding claims. Since he does not adopt one of them as his basic account, I will not discuss these modifications here.

The Problem of Triviality. This worry concerns the substantiality of Maguire's autonomy thesis. I will argue that MA and CMA fail to satisfy the desiderata in virtue of their insensitivity to genuine counterexamples and their compatibility with certain forms of naturalism. To see why recall that DISJUNCTION, where $D \vee N$ is grounded solely by descriptive $D$, is a spurious counterexample. Maguire's autonomy thesis is not falsified by the example because $D \vee N$ counts as descriptive in those cases. The question arises why we should accept that result. One answer would be that it must be descriptive in order to make this example compatible with the autonomy thesis. This answer would be ad hoc. Moreover, it would render the metaphysical autonomy thesis trivially true.

The answer that Maguire seems to suggest is that grounding preserves the taxonomic status. ${ }^{37}$ Again, in order for the autonomy thesis to be substantial this claim needs to be motivated independently. This can perhaps be done by referring to a common way to articulate grounding claims: The grounded is nothing over and above its grounds. So, in this case $D \vee N$ would be nothing over and above $D$. This explanation is independent from the truth of the autonomy thesis. The plausibility of the explanation depends on its generality. I will argue that if the explanation generalizes, then Maguire's autonomy thesis is insensitive to genuine counterexamples and, more generally, compatible with a naturalist view of act utilitarianism. Thus, the account does not satisfy the third and fourth desideratum. Assume that the fact that act $a$ maximizes overall pleasure grounds the fact that $a$ is morally obligatory. We assume that there are no other grounds for the fact that $a$ is morally obligatory. According to the preservation principle, this fact counts as descriptive. Therefore, the example is compatible with Maguire's autonomy thesis.

Maguire would probably object that this fact is a paradigmatic normative fact and that we therefore should reject his autonomy thesis in this case. But if grounding preserves the taxonomic status of facts in a way that is independent of his autonomy thesis, then I cannot see why paradigmatic examples of normative facts should be excluded from this general preservation principle. This might not be a decisive reason to reject Maguire's thesis as a form of autonomy thesis: The compatibility results from the trivial truth of the autonomy thesis in those cases. If the naturalist view of act utilitarianism and the preservation principle is correct, then there are no particular normative facts. Hence, Maguire's autonomy thesis is trivially true. But this result fails to do justice to the fourth desideratum according to which a substantial autonomy

\footnotetext{
36 See (Fine 2012a) for problems of defining non-factive grounding in terms of factive grounding.

37 See (Maguire 2015, p. 190). However, we will see that the preservation principle is incompatible with some forms of non-reductive naturalism. For non-reductive naturalists might want to say that normative facts are fully grounded in (and only in) descriptive facts, and yet normative.
} 
thesis is incompatible with metaethical views which take the normative domain to be fully generated by the descriptive domain.

Let us take stock and sum up the results so far. Maguire's autonomy thesis entails implausible taxonomic consequences. For it treats relevantly similar cases different. MA and CMA handle spurious counterexamples either in an ad hoc way that trivializes the autonomy thesis or by appeal to a more general preservation principle that renders the autonomy thesis insensitive to genuine counterexamples and competitive views like naturalism.

\subsection{Normatively relevant grounding}

Now I will turn to my own proposal. The lesson that can be drawn from the spurious counterexamples is that the autonomy thesis should be formulated in terms of normative relevance. The mechanism that produces the spurious counterexamples exploits features of disjunctions. There is at least one purely descriptive way for the normative proposition to be true. The descriptive propositions that do the grounding work are relevant only to the descriptive ways for the normative proposition to be true; they are irrelevant to those ways for the normative proposition to be true that are responsible for its normative status. Hence, the Relevant Autonomy Thesis states:

Relevant Autonomy Thesis (RAT) No normative proposition is fully grounded ${ }^{38}$ in descriptive propositions in a normatively relevant way.

The relevant autonomy thesis is formulated in terms of normatively relevant grounding. The next step is to make this concept formally precise. The approach is based on truthmaker semantics. Grounding ensures that the grounds are relevant to the grounded proposition in some way, while it does not ensure that the grounds are relevant to the the grounded proposition in a normative way, i.e. to the normative parts of the grounded proposition. Accordingly, we must identify those parts ${ }^{39}$ of a proposition that are responsible for its normative status. We may take the part of a normative proposition $N$ that is responsible for $N$ 's normative status to be all its normative truthmakers. Recall that $S^{N}$ is the set of all normative states. So, we consider the intersection of the normative proposition $N$ and the set of all normative states $S^{N}:\left.N\right|_{S^{N}}:=N \cap S^{N}$. Intuitively, these are all the normative ways for $N$ to be true. I will use the term normative realization to refer to $\left.N\right|_{S^{N}}$. We now have the means to make the concept of normatively relevant grounding precise.

NORMATIVELY RELEVANT GROUNDING $P_{1}, P_{2}, \ldots$ fully ground $Q$ in a normatively relevant way $\Leftrightarrow{ }_{D f} . P_{1}, P_{2}, \ldots$ fully ground $\left.Q\right|_{S^{N}}$.

Note that normatively relevant grounding is not supposed to be a different kind of grounding. The relevance constraint simply excludes the possibility of certain sorts of

\footnotetext{
38 I assume the non-factive conception of grounding according to which true grounding claims do not entail that the relata are true. I will use the non-factive concept of ground throughout the rest of the paper. Otherwise, it will be indicated.

39 I do not use the notion of propositional part in the sense Fine uses it in Fine (2017b). My way of using it corresponds to what Fine calls disjunctive parts.
} 
grounding claims. ${ }^{40}$ With this concept of normatively relevant grounding at hand, we can make the RAT formally precise:

(PROP-RAT) No normative proposition $Q$ is such that its normative realization $\left.Q\right|_{S^{N}}$ is fully grounded in descriptive propositions.

Since the present account is based on truthmaker semantics, it is worth explicitly stating the thesis that corresponds to the RAT on the level of states. To this aim, we need truthmaker semantics for statements of ground. A promising candidate is Fine (2012b)'s approach. He defines the notion of weak full ground as follows:

Weak full ground $(\leq) P_{1}, P_{2}, \ldots$ weakly fully ground $Q$ iff $s_{1} \sqcup s_{2} \sqcup \ldots$ makes $Q$ true whenever $s_{1}, s_{2}, \ldots$ make $P_{1}, P_{2}, \ldots$ true, respectively. ${ }^{41}$

Note that, in contrast to our more intuitive understanding of ground, weak full ground is reflexive. This is, for any $P$ we have $P \leq P{ }^{42}$

One might object that the RAT thus explicated is trivially true. To see why, note that if we adopt Fine's truthmaker semantics for ground and assume that no normative state is identical to a descriptive state, then by definition it is impossible for a descriptive proposition, i.e. a set of descriptive truthmakers, to ground a normative realization of a proposition, i.e. the maximal normative subset of a proposition. Since no set of descriptive states is a subset of a set of normative states, no descriptive proposition grounds a normative proposition. But this result would be due to our semantics and our taxonomy. ${ }^{43}$ The truth of the RAT would not depend on the nature of normativity, and thus would violate (D1).

This illustrates that our semantics together with our assumptions about the taxonomy is not sufficiently neutral. To be clear, the RAT is not supposed to be neutral. It makes a substantial claim that is incompatible with e.g. naturalism. However, the semantical framework that we use should not prejudge the truth of the RAT.

To see that the semantical framework, as it stands, is incompatible with naturalism, consider a naturalistic view of act utilitarianism. Let $D$ be the proposition that act $a$ maximizes pleasure and $d$ the descriptive state that makes the proposition true. Let $N$ be the proposition that act $a$ is morally required and $n$ the normative state that makes the proposition true. According to the view under consideration, $D$ grounds $N$. However, since $d$ is descriptive and $n$ is normative they are distinct. Thus, it is not the case that $\{d\}$ is a subset of $\{n\}\left(\left.D \nsubseteq N\right|_{S^{N}}\right)$ and hence it is not the case that $D$ (weakly) grounds $\left.N\right|_{S^{N}}$.

In response to this objection, we adopt a truthmaker-based semantics for ground that is sufficiently neutral. Krämer (2021) suggests the following modification of Fine's truthmaker semantics for ground:

\footnotetext{
40 The autonomy thesis is a negative claim. We will discuss the question of what fully grounds normative propositions in a normatively relevant way in Sect. 4.

${ }^{41}$ See (Fine 2012b, p. 9). Fine uses the term 'verifier' instead of 'truthmaker'. For reasons of uniformity, I changed the terminology.

42 See (Fine 2012a) for a discussion of how to define strict full ground in terms of weak full ground.

${ }^{43}$ Recently, Krämer (2021) points out that Fine's truthmaker-based semantics for ground rules out seemingly possible grounding structures.
} 
Weak full ground* $\left(\leq^{*}\right) P_{1}, P_{2}, \ldots \leq \leq^{*} Q$ iff $s_{1} \sqcup s_{2} \sqcup \ldots$ generates some truthmaker $t$ of $Q$ whenever $s_{1}, s_{2}, \ldots$ make $P_{1}, P_{2}, \ldots$ true, respectively. ${ }^{44}$

A state $s$ generates a state $t$ iff either $s$ is identical to $t$ or the proposition made true by $s$ and only $s$ strictly fully grounds the proposition made true by $t$ and only $t$ (Krämer 2021, p. 48). ${ }^{45}$ The definition illustrates that there is an intimate connection between generation on the level of states and grounding on the level of propositions. Generation can be described as the relationship of weak grounding on the level of states. In the naturalist example, $d$ generates $n$ since we assumed that $D$ grounds $N$ and that $d$ and $n$ are the sole truthmakers of $D$ and $N$, respectively.

One might be worried that the semantical framework is still not sufficiently neutral because it cannot account for views that assume multiple realization. Consider for example the normative proposition that action $a$ promotes the good. Assume that this proposition can be multiply realized in descriptive ways, e.g. $a$ promotes pleasure, $a$ promotes knowledge, $a$ promotes friendship, etc. If we were to say that the corresponding descriptive states are exact truthmakers of the proposition that $a$ promotes the good, then this proposition would be descriptive. So in order to preserve the normative-descriptive divide on the level of propositions we shall say that the proposition has a normative truthmaker. Formally, we can make room for this multiple realizer view by modeling their claim as follows: the descriptive states that correspond to the descriptive realizers generate a normative truthmaker for the normative proposition that $a$ promotes the good. Thus, our semantical framework does not prejudge the truth of the RAT. Within this neutral framework we can formulate the autonomy thesis on the level of states such that the result is equivalent to the RAT: ${ }^{46}$

(STATE-RAT) No descriptive state generates a normative state.

In the next section, we will see that the RAT is a formally precise substantial autonomy thesis. It thereby provides the groundwork for a comprehensive defense of the autonomy of the normative domain.

\section{A substantial claim}

The aim of this section is to show that the RAT satisfies the outlined desiderata.

Desiderata (D2)-(D4). To see whether the desiderata are satisfied, we survey whether the RAT is immune to spurious counterexamples (D2), sensitive to genuine putative counterexamples (D3), and incompatible with certain metaethical views (D4).

\footnotetext{
44 See (Krämer 2021, p. 48). Krämer uses the term 'verifier' instead of 'truthmaker'. For reasons of uniformity, I changed the terminology.

45 Notice that the requirement that $s$ and $t$ are unique truthmakers of the propositions in question is only relevant to the question of whether $s$ generates $t$. Grounds and grounded proposition might have several truthmakers.

46 Proof: I show that $\neg$ (PROP-RAT) is equivalent to $\neg$ (STATE-RAT). We assume that the set of descriptive states $S^{D}$ is closed under fusion. $\neg$ (PROP-RAT) $\Leftrightarrow$ Some descriptive $P_{1}, P_{2}, \ldots$ fully ground $\left.Q\right|_{S^{N}}$ for normative $Q . \Leftrightarrow d_{1} \sqcup d_{2} \sqcup \ldots$ generates some truthmaker $n$ of $\left.Q\right|_{S^{N}}$ whenever $d_{1}, d_{2}, \ldots$ make $P_{1}, P_{2}, \ldots$ true, respectively. $\Leftrightarrow$ A descriptive state, namely $d_{1} \sqcup d_{2} \sqcup \ldots$, generates a normative state $n \Leftrightarrow \neg$ (STATERAT).
} 
First, we will go through the spurious counterexamples mentioned above and see how the RAT can handle those cases. We can assume without loss of generality that in DisJunction $D=\{d\}$ and $N=\{n\}$ where $d$ is a descriptive state and $n$ is a normative state. $\left.(D \vee N)\right|_{S^{N}}=\{n, d \sqcup n\}^{47}$ is not fully grounded by $D$ because $d$ does not generate any state that makes $\left.(D \vee N)\right|_{S^{N}}$ true. This is plausible since in general $D$ is irrelevant to $N .^{48}$ Hence, $D$ does not ground $D \vee N$ in a normatively relevant way or if it does, it is a genuine counterexample.

In ABSENCE, we assume that Sarah's non-existence, $\bar{e}$, is the sole truthmaker of Sarah does not exist. The normative proposition There is no obligation of Sarah to donate is made true by Sarah's non-existence or by Sarah's permission to keep her money, $p$. If we assume that propositions are closed, i.e. that any fusion of truthmakers is a truthmaker of the proposition in question, then the proposition is also made true by the impossible state $\bar{e} \sqcup p$. Hence, There is no obligation of Sarah to donate $\left.\right|_{S^{N}}=$ $\{p, \bar{e} \sqcup p\}$ is not fully grounded by Sarah does not exist because $\bar{e}$ does not generate any state that makes There is no obligation of Sarah to donate $\left.\right|_{S^{N}}$ true. Hence, Sarah does not exist does not ground There is no obligations of Sarah to donate in a normatively relevant way.

In general, whenever a proposition $N$ has at least one descriptive and one normative truthmaker, then this proposition is i) normative in virtue of the normative truthmaker and ii) if at least one of the descriptive truthmakers obtains, fully grounded by a descriptive proposition, namely the proposition that is identified with the singleton of one of the obtaining descriptive truthmakers of $N$. Such cases do not undermine the RAT because it focuses on the normative realization of a proposition $\left.N\right|_{S^{N}}$. It requires that the grounds are normatively relevant to the grounded proposition.

One might ask whether the success of the RAT is restricted to disjunctive cases. Some spurious counterexamples from the literature do not seem to share the disjunctive structure. I will point out that the RAT can handle those cases as well. Consider a ground-based version of an example in Prior (1960):

UNDERTAKER The descriptive proposition that all undertakers are Church officers grounds the proposition that if all Church officers ought to be reverent, then all undertakers ought to be.

In order to analyze the example, we need to identify the truthmakers of the conditional. In general, it is not easy to tell which states make a conditional true. One possibility is to treat the grounded proposition as material conditional. However in this case, it seems more plausible that the conditional is supposed to state a relevant connection between the antecedent and the consequent. I lack the space to introduce the truthmaker semantics for the conditional in relevance logic. ${ }^{49}$ Instead, I will go through the intuitive idea behind the semantics and show that the RAT can account for this example. Which states make the proposition that if all Church officers ought to be reverent, then all undertakers ought to be true? Arguably, every state that bridges the

\footnotetext{
47 I am assuming that disjunctions are closed under fusion.

48 If $d$ generates $n$, then this case would be a genuine counterexample. But this is the desired result, since $D$ then would be normatively relevant to the normative realization of $D \vee N$. I will discuss this case in detail in the Sect.5.

49 See (Fine 2014).
} 
gap between the truthmakers of the antecedent and the truthmakers of the consequent. The state $s$ makes the conditional true iff every fusion of $s$ and a truthmaker of the antecedent contains a truthmaker of the consequent. In our case, two different kinds of states satisfy this condition:

D-State the state that all undertakers are Church officers;

$\mathbf{N}$-State the state that Church officers and undertakers have the same obligations.

Both states bridge the gap between truthmakers of the antecedent and truthmakers of the consequent. Note that D-State is a descriptive state. It also makes the proposition that if all Church officers wear shoes, then all undertakers do true. In contrast, $\mathbf{N}$ State is a normative state. Consider the proposition that if police officers ought to be reliable, then firemen ought to be. The state that firemen and police officers have the same obligations is normative and does not depend on the subset relation between the set of police officers and firemen. UNDERTAKER is no instance of normatively relevant grounding because the normative realization of the conditional is not grounded in the descriptive proposition. The RAT handles those cases similar to the examples that were discussed before: It shows that the descriptive ground is not relevant to the normative part of the grounded proposition. It is an additional advantage of the RAT that it illustrates that spurious counterexamples are ultimately based on the same mechanism. 50

To show that the RAT is sensitive to genuine putative counterexamples and incompatible with naturalist metaethical views, assume that a naturalist view of act utilitarianism is true. In other words, assume that particular normative propositions are fully grounded in descriptive, i.e. non-normative propositions. Assume that e.g. the following is true: The descriptive proposition that act $a$ maximizes overall pleasure $(=\{d\})$ fully grounds the proposition that $a$ is morally obligatory $(=\{n\})$. In this case, the descriptive proposition is fully relevant to the normative proposition. To satisfy (D3) the RAT needs to be incompatible with this genuine counterexample.

Indeed, the RAT is incompatible with the example and thus incompatible with this form of naturalism. Note, that it is the RAT that is incompatible with naturalism rather than our semantics which is supposed to be neutral. The STATE-RAT which is equivalent to the RAT shows this result. According to the STATE-RAT, no descriptive state generates a normative state. However, in the naturalist case, the truthmaker of the proposition that act $a$ maximizes overall pleasure would generate a normative state that makes the proposition that $a$ is morally obligatory true. For the former descriptive proposition strictly grounds the latter normative proposition. Therefore, a descriptive state generates a normative state. Thus, the STATE-RAT would be false. The same

\footnotetext{
50 An anonymous referee raised the question whether the RAT can handle an example that is attributed to Rosen. The descriptive proposition that Sue stands in front of children grounds the normative proposition that Sue ought not to flurg. To flurg is defined as doing something you ought not to do in front of children. To identify the truthmakers of the grounded proposition is a complex task because of the complex structure of the proposition. However, the strategy is similar to the previously discussed cases. In order for this example to be an instance of grounding, one either must agree that the descriptive state that Sue stands in front of children is a truthmaker for the grounded proposition or that this state generates some normative truthmaker of the grounded proposition, e.g. that Sue ought not to smoke. It is unclear why a proponent of the autonomy thesis should accept the second option. The first option can be handled by the RAT.
} 
holds for the multiple realizer case because the normative truthmaker is generated by descriptive states. Thus, the RAT satisfies the third and fourth desideratum.

(D1), (D5), and the metaethical terrain. The previous considerations raise the more general question of how the RAT can be located in the metaethical terrain. ${ }^{51}$ Which views can accept the RAT? In the rest of this section, we consider realist and anti-realist views and see whether the RAT non-trivially divides the metaethical terrain.

The RAT is a ground-theoretic thesis about the normative domain. It satisfies (D1) in virtue of the fact that it makes a claim about the nature of the normative domain. As such it is naturally linked to normative realism according to which there are normative facts or propositions. In the realist camp, naturalist views roughly state that normative facts ultimately concern natural things. Independently of more concrete formulations, naturalism seems to be incompatible with the explanatory insufficiency of the descriptive realm. This is why we introduced the fourth desideratum (see above Sect. 1) which requires that the RAT is incompatible with these metaethical views.

In contrast, the spirit of non-naturalism seems to fit the RAT. Non-naturalism is sometimes characterized in terms of the autonomy or the sui generis status of normative facts. Rosen (2017) explicates non-naturalism in terms of ground: (some) atomic normative facts are not fully metaphysically grounded in descriptive facts. Under this explication non-naturalism dovetails with the RAT. This leaves us with the question of how normative facts are grounded according to these views. While a detailed discussion is beyond the scope of this paper, I will briefly point out some options. First, nonnaturalists can hold that normative facts are partially grounded in descriptive facts but fully grounded in descriptive facts together with normative partial grounds like moral laws or principles. ${ }^{52}$ Second, non-naturalists can endorse that normative facts are fully normatively rather that fully metaphysically grounded in non-normative facts. ${ }^{53}$ For the RAT is stated in terms of metaphysical ground. Third, non-naturalists might employ recent accounts of merely partial ground according to which some facts have partial grounds but fail to be fully grounded by any collection of facts. ${ }^{54}$ Yet, there are variants of non-naturalism that are incompatible with the RAT. Consider the view that normative facts are different in kind from, yet fully grounded in, descriptive facts. The nature of the normative grounded fact does not make contact, we might say, with the nature of the grounds. Rosen (2010) calls these connections Moorean connections and argues that we cannot rule them out on principled grounds. ${ }^{55}$ This form of nonnaturalism is incompatible with the RAT. ${ }^{56}$ I take this result to show that generally, the (ground-theoretic and logical ${ }^{57}$ ) autonomy theses do not prejudge which metaethical views must fall on which side of the autonomy debate. If it turns out that some non-

\footnotetext{
51 Thanks to an anonymous referee for asking me to address this question.

52 See (Rosen 2017). For critical discussion and reply see (Berker 2019) and (Fogal and Risberg 2020).

53 See (Bader 2017).

54 See (Leuenberger 2020) and (Trogdon and Witmer 2021).

55 A serious doubt concerning this view has been raised by Bader (2017). He shows that it requires to individuate facts even finer than by ground-theoretic equivalence.

56 In contrast to Maguire's thesis, however, the RAT does not commit this view to the implausible taxonomic consequence that the grounded fact is descriptive.

57 If grounds necessitate what they ground, Moorean connections falsify autonomy theses in terms of entailment.
} 
naturalist cannot accept the sense of autonomy specified by the RAT, then this shows that not all non-naturalists are autonomists.

On the other hand, consider anti-realist views: One might think that these views, by design, cannot non-trivially accept the autonomy of the normative domain. ${ }^{58}$ For they deny that there are normative facts or true propositions. In the rest of this section, I argue that anti-realist views can nevertheless take a non-trivial stance on the autonomy debate. First, consider error theories. Since error theorists think that there are no true (atomic) normative statements at all, it seems trivial for them that one cannot get a normative truth from a descriptive truth. However, that there are no (atomic) normative truths does not entail the RAT. For the RAT states that normative propositions-true or not-are not grounded in a normatively relevant way. The concept of ground that is used is non-factive. Thus, the RAT is already false if for example a false normative proposition is fully (non-factively) grounded in descriptive propositions in a normatively relevant way. An error theorist would plausibly reject this case, and thus can accept the RAT in a non-trivial way. For if normative propositions were fully non-factively grounded in descriptive propositions, they would arguably be metaphysically continuous with descriptive propositions. ${ }^{59}$ The problematic nature of normative propositions as it is assumed by error theorists would disappear. Second, consider non-cognitivist views. Roughly, non-cognitivists claim that normative judgements do not predicate properties. So strict forms of non-cognitivism are plausibly not concerned with any autonomy debate. However, hybrid forms of non-cognitivism acknowledge that normative judgements can have propositional content. In particular, these views are supposed to account for the truth of complex normative judgements and normative reasoning. ${ }^{60}$ To see that these views are concerned with the autonomy debate, consider an example discussed in Schroeder (2009): From 'Jeremy said that stealing is wrong' and 'Everything that Jeremy said is true' we can infer 'Stealing is wrong'. Schroeder argues that to secure the argument's validity, hybrid forms of expressivism are committed to the claim that 'Everything that Jeremy said is true' expresses a desire-like attitude. That is, one of the premises must be normative. Similar considerations might apply to a ground-theoretic autonomy thesis. It would be interesting to see whether these views can accept judgements of the form $N$ fully because $D$ where $D$ is descriptive, $N$ is normative, and 'because' is a sentential operator. ${ }^{61}$

To sum up, the RAT provides us with a substantial interpretation of the autonomy of the normative domain that is compatible with different realist views, but incompatible with naturalist views. In addition, although a ground-based autonomy debate most naturally applies to realist views, anti-realists can interpret the RAT in a metaphysically less significant way.

\footnotetext{
58 Thanks to an anonymous referee for raising this question.

59 See (McPherson 2012).

60 This embedding problem is often called the Frege-Geach problem. For an overview see (van Roojen 2018).

61 For a recent discussion of how quasi-realist can account for the relevant because-statements see (Berker 2020).
} 


\section{Comparing the two ground-theoretic approaches}

Finally, I will compare the RAT to the ground-theoretic approach in Maguire (2015). I show how the relevant autonomy thesis avoids the concerns I have raised against Maguire's account and thereby generates a decisive advantage: it provides us with a substantial autonomy thesis that is sensitive to genuine counterexamples.

The Problem of Contingentism. I have argued that Maguire's account entails implausible taxonomic consequences. Recall that according to his view the fact that it is raining or murder is morally wrong and the fact that it is raining or charity is morally wrong differ in their taxonomic status. However, both disjunctive facts seem to be partly about rainy weather and partly about moral wrongness. Furthermore, non-obtaining facts are not subject to Maguire's autonomy thesis at all. The RAT is not subject to this concern. The RAT entails an essentialist account. Essentialism takes the taxonomic status of propositions to be essential to them. The taxonomic status is determined by the truthmaker-based content of a proposition. Therefore, the RAT applies to all propositions independently of their truth-value.

The Problem of Triviality. I have argued that the preservation principle trivializes Maguire's autonomy thesis. The RAT avoids this worry because there are independent criteria that determine the normative and the descriptive domain. They allow the characterization of a proposition as normative even if there are only descriptive full grounds. As a result, the taxonomy is neutral between competitive metaethical views. In contrast, the RAT turns out to be false, if there is a descriptive full ground of the normative realization of a proposition.

This illustrates another important advantage of the RAT over Maguire's autonomy thesis: The RAT distinguishes between cases where the full descriptive ground of a normative proposition is relevant only to the descriptive parts of the grounded proposition and those cases where it is (additionally) relevant to the normative parts. The latter but not the former cases constitute genuine counterexamples. Maguire's autonomy thesis is compatible with both kinds of cases, while the RAT is just compatible with the former cases and thus satisfies the desiderata. To see why, we consider two examples. Consider an instance of DISJUNCTION: $R$ grounds $R \vee M . R$ is the descriptive proposition that it is raining and $M$ is the normative proposition that murder is wrong. Note that the fact that it is raining is only relevant to the descriptive parts of the disjunction and irrelevant to the normative parts of the disjunction. Both the RAT and Maguire's autonomy thesis are compatible with this spurious counterexample. Now, we consider the descriptive proposition $H$ that murder causes harm. Again, we can produce an instance of DisJUNCTION: $H$ grounds $H \vee M$. Suppose it turns out to be true that the fact that murder causes harm fully grounds the fact that murder is morally wrong. Accordingly, the fact that murder causes harm is relevant to the normative parts of the disjunction, namely $M$. The RAT is incompatible with this genuine example since the normative realization of $H \vee M$ is fully grounded in $H$ by assumption. Thus, $H$ fully grounds $H \vee M$ in a normatively relevant way. To sum up, if the fact that murder causes harm fully grounds the fact that murder is morally wrong, then the RAT is false. In contrast, Maguire's autonomy thesis is compatible with this genuine example. This is because the second example is an instance of DISJUNCTION. If there is a normative ground for $H \vee M$, then the disjunction is not grounded just in descriptive 
facts and if $H$ is the sole ground, then $H \vee M$ is descriptive. At least as far as I can see, there is no obvious way to achieve this result for Maguire's approach.

The RAT is not subject to the concerns that have been raised against Maguire. Therefore, it is a promising way to provide us with a substantial understanding of the abstract autonomy claim.

\section{Conclusion}

The debate about the autonomy of the normative domain shows that it is difficult to give a precise formulation of the abstract autonomy claim that provides us with a substantial thesis about the nature of the normative domain. I have introduced five desiderata for a substantial autonomy thesis which require that the thesis is falsified by genuine in contrast to spurious counterexamples and that the thesis is incompatible with certain naturalist claims. I have offered an explanation of the mechanism behind spurious counterexamples: Descriptive propositions are not relevant to those propositional parts of a normative proposition that are responsible for its normative status-that is, its normative content. The relevant autonomy thesis, the RAT, states that no normative proposition is grounded in descriptive propositions in a normatively relevant way. The truthmaker-based interpretation of normative relevance avoids those spurious examples, while being sensitive to genuine putative counterexamples. The present account thus lays the groundwork for a comprehensive defense of the RAT by clarifying the assumptions it will require. Furthermore, it provides us with a further relevant perspective on different metaethical views. The RAT is a precise formulation of a substantial interpretation of the abstract autonomy claim that avoids commitments to implausible taxonomic consequences.

Acknowledgements The research for this paper was funded by the Deutsche Forschungsgemeinschaft through the Emmy Noether grant on Relevance (KR 4516/2-1), and I gratefully acknowledge the support. Earlier versions of the material were presented at the GAP PhD-workshop on Mathematical Philosophy (2019), at the ECAP.10 (2020) conference, and at the conference on Relevance, Grounding, and Normativity (2020) in Hamburg. I thank all members of the audiences for their helpful comments and criticisms. Special thanks are due to Stephan Krämer, Martin Glazier, and Stefan Roski for their extensive and especially useful feedback. Finally, I would like to thank two anonymous referees for this journal for very helpful comments and suggestions which have resulted in a number of improvements.

Funding Open Access funding enabled and organized by Projekt DEAL.

\section{Declarations}

Conflict of interest I declare that I have no conflict of interest.

Open Access This article is licensed under a Creative Commons Attribution 4.0 International License, which permits use, sharing, adaptation, distribution and reproduction in any medium or format, as long as you give appropriate credit to the original author(s) and the source, provide a link to the Creative Commons licence, and indicate if changes were made. The images or other third party material in this article are included in the article's Creative Commons licence, unless indicated otherwise in a credit line to the material. If material is not included in the article's Creative Commons licence and your intended use is not permitted 
by statutory regulation or exceeds the permitted use, you will need to obtain permission directly from the copyright holder. To view a copy of this licence, visit http://creativecommons.org/licenses/by/4.0/.

\section{References}

Asay, J. (2013). Truthmaking, metaethics, and creeping minimalism. Philosophical Studies, 163(1), 213232. https://doi.org/10.1007/s11098-011-9808-0.

Bader, R. M. (2017). The grounding argument against non-reductive moral realism. Oxford Studies in Metaethics, 12, 904-936.

Berker, S. (2019). The explanatory ambitions of moral principles. Noûs, 53(4), 904-936.

Berker, S. (2020). Quasi-dependence. Oxford Studies in Metaethics, 15, 195-218.

Brown, C. (2014). Minding the Is-Ought Gap. Journal of Philosophical Logic, 43(1), 53-69.

Clipsham, P. (2015). Developing a post-prior taxonomy of ethical sentences. Philosophia, 43(3), 801-820.

Enoch, D. (2010). How objectivity matters. Oxford Studies in Metaethics, 5, 111-52.

Fine, K. (2012a). Guide to ground. In F. Correia \& B. Schnieder (Eds.), Metaphysical grounding (pp. 37-80). Cambridge University Press.

Fine, K. (2012b). The pure logic of ground. The Review of Symbolic Logic, 5(1), 1-25. https://doi.org/10. 1017/S1755020311000086.

Fine, K. (2014). Truth-maker semantics for intuitionistic logic. Journal of Philosophical Logic, 43(2-3), 549-577. https://doi.org/10.1007/s10992-013-9281-7.

Fine, K. (2017a). A theory of truthmaker content I: Conjunction, disjunction and negation. Journal of Philosophical Logic, 46(6), 625-674.

Fine, K. (2017b). A theory of truthmaker content II: Subject-matter, common content, remainder and ground. Journal of Philosophical Logic, 46(6), 675-702.

Fine, K. (2021). Truthmaking and the is-Ought Gap. Synthese, 198, 887-914. https://doi.org/10.1017/ s11229-018-01996-8.

Fogal, D., \& Risberg, O. (2020). The metaphysics of moral explanations. Oxford Studies in Metaethics, 15. https://doi.org/10.1093/oso/9780198859512.003.0008.

Gibbard, A. (2012). Meaning and normativity. UK: Oxford University Press.

Hume, D. (1978). A treatise of human nature (2nd ed.). New York: Oxford University Press.

Jago, M. (2020). Truthmaker semantics for relevant logic. Journal of Philosophical Logic, 49, 681-702. https://doi.org/10.1007/s10992-019-09533-9.

Krämer, S. (2021). Singular troubles with singleton socrates. Philosophy and Phenomenological Research, 103(1), 40-56. https://doi.org/10.1111/phpr.12692.

Leuenberger, S. (2020). The fundamental: Ungrounded or all-grounding? Philosophical Studies, 177(9), 2647-2669. https://doi.org/10.1007/s11098-019-01332-x.

Maguire, B. (2015). Grounding the autonomy of ethics. Oxford Studies in Metaethics, 10, 188-215. https:// doi.org/10.1093/acprof:oso/9780198738695.003.0008.

Maitzen, S. (2010). Moral Conclusions from Non-Moral Premises. In C. Pigden (Ed.), Hume on Is and Ought (pp. 290-309). Palgrave MacMillan.

McPherson, T. (2012). Ethical non-naturalism and the metaphysics of supervenience. Oxford Studies in Metaethics, 7, 205-243. https://doi.org/10.1093/acprof:oso/9780199653492.003.0007.

Oddie, G. (2018). Non-naturalist moral realism, autonomy and entanglement. Topoi, 37(4), 607-620.

Pigden, C. R. (1989). Logic and the autonomy of ethics. Australasian Journal of Philosophy, 67(2), 127-151.

Pigden, C. R. (Ed.). (2010). Hume on Is and Ought. Palgrave Macmillan.

Prior, A. (1960). The autonomy of ethics. Australasian Journal of Philosophy, 38(3), 199-206.

Rosen, G. (2010). Metaphysical dependence: Grounding and reduction. In B. Hale \& A. Hoffmann (Eds.), Modality: Metaphysics, logic, and epistemology (pp. 109-36). Oxford: Oxford University Press.

Rosen, G. (2017). Metaphysical relations in metaethics. In T. McPherson \& D. Plunkett (Eds.), (pp. 151169). Routledge: The Routledge Handbook of Metaethics.

Russell, G., \& Restall, G. (2010). Barriers to Implication. In C. R. Pigden (Ed.), Hume on Is and Ought (pp. 243-259). Palgrave MacMillan.

Rynin, D. (1957). The autonomy of morals. Mind, 66(263), 308-317.

Schroeder, M. (2009). Hybrid expressivism: Virtues and vices. Ethics, 119(2), 257-309.

Singer, D. J. (2015). Mind the is-ought gap. Journal of Philosophy, 112(4), 193-210. 
Trogdon, K. (2013). An introduction to grounding. In M. Hoeltje, B. Schnieder \& A. Steinberg (Eds.), Varieties of dependence (pp. 97-122). Munich, Germany: Philosophia Verlag.

Trogdon, K., \& Witmer, D. (2021). Full and partial grounding. Journal of the American Philosophical Association, 7(2), 252-271. https://doi.org/10.1017/apa.2020.26.

van Roojen, M. (2018). Moral cognitivism vs. non-cognitivism. In E. N. Zalta (Ed.), The Stanford Encyclopedia of Philosophy (Fall 2018). Metaphysics Research Lab: Stanford University.

Williams, B. (1985). Ethics and the limits of philosophy. Harvard University Press.

Woods, J. (manuscript). On vacuous grounding: The case study of ethical autonomy.

Publisher's Note Springer Nature remains neutral with regard to jurisdictional claims in published maps and institutional affiliations. 